\title{
Diffusion-weighted MRI to Evaluate Immediate Response to Irreversible Electroporation in a Rabbit VX2 Liver Tumor Model
}

Short title: DW MRI to evaluate response of IRE in VX2 liver tumor

\begin{abstract}
Purpose: To evaluate the feasibility of diffusion-weighted (DW) MRI (DW-MRI) for quantitative measurement of responses following irreversible electroporation (IRE) procedure in rabbit liver tumor model.

Materials and Methods: Twelve rabbits underwent ultrasound-guided VX2 tumor implantation in the left medial and left lateral liver lobes. The tumors in the left medial lobe were treated with IRE, while those in the left lateral lobe served as internal controls. DWI were performed before and immediately post-IRE. Then tumors were harvested for histopathological staining. The apparent diffusion coefficient (ADC) and changes in ADC $(\triangle A D C)$ were calculated from DWI. Tumor apoptosis index were assessed by terminal deoxynucleotidyl transferase dUTP nick end labeling (TUNEL) staining. These measurements from DWI and histopathology were compared between untreated and treated tumors.
\end{abstract}

Results: The $A D C$ values, $\triangle A D C$, and apoptosis index showed statistically significant difference between treated and untreated tumors $(P<0.05$ for all). The ADC values was 
higher in the treated tumors than in the untreated tumors $(1.08 \pm 0.15$ vs $0.88 \pm 0.19 \times$ $\left.10^{-3} \mathrm{~mm}^{2} / \mathrm{s}, P=0.042\right)$.

Conclusions: DWI can be used to quantitatively evaluate treatment response in liver tumors immediately after IRE procedure

Keywords: Irreversible electroporation, Ablation, Liver tumors, Apparent diffusion coefficient, Magnetic resonance imaging

\section{INTRODUCTION}

While thermal ablation has become common used for treatment of unresectable primary and metastatic liver tumors, these methods have also demonstrated several limitations (the risk of collateral damage to vascular, biliary or other heat-sensitive structures; less effective in lesions proximity to major blood vessels) that limit efficacy in the clinical setting ${ }^{1}$. Irreversible electroporation (IRE) is a ablation technology that overcomes many of the primary limitations of thermal ablation approaches ${ }^{2}$. IRE can efficaciously ablate tumor around the hepatic hilum and create uniform ablation zones around hepatic veins $^{3,4}$. Additional advantages include generation of a tumor specific immunological reaction ${ }^{5,6}$. These benefits make IRE an attractive potential approach for the treatment of liver tumors. However, imaging approaches previously demonstrated to be highly effective for early detection of response to thermal ablation may be inappropriate and ineffective for detecting acute response to IRE treatment. The development of non- 
invasive imaging techniques for accurate assessment of IRE treatment will be useful for effective clinical application ${ }^{7,8}$.

Magnetic resonance imaging (MRI) has been commonly used for the assessment of immediate response after IRE ablation of liver tumors ${ }^{9,10}$. However, relatively few reports evaluate MR findings for intraprocedural monitoring and assessing therapy effects after IRE in liver tumor ${ }^{2,7-9}$. Contrast-enhanced MRI is the most widely used technique in these studies $^{7}$. Diffusion-weighted imaging (DWI) MRI has proven to be very sensitive for the detection of cell death and tissue damage ${ }^{11}$. IRE produces rapid, dramatic changes within the tumor microenvironment. These microstructural changes remove barriers to watermobility ${ }^{1,10,12}$. DWI measurements of these acute alterations to water-mobility may be ideal for in vivo assessment of IRE response. The apparent diffusion coefficient (ADC), the most common quantitative parameter extracted from DWI, can characterize tumor microstructure because water diffusion is affected by tumor cellularity, necrosis, and other characteristics $^{13-16}$. Several previous studies have demonstrated that DWI can evaluate treatment response in tumor as early as 24 hours, which is prior to morphological changes $^{14,16}$. Hence, DWI may also effectively evaluate the early response of liver tumor to IRE treatment in this study. The purpose of this study was to evaluate the feasibility of DWI for quantitative assessment of treatment response immediately after IRE procedure of liver tumor in a rabbit model.

\section{MATERIALS AND METHODS}


All studies were approved by the institutional animal care and use committee of \#\#\#\#\#\#\#\# (IS\#\#\#\#\#\#) and performed in accordance with National Institutes of Health guidelines.

\section{Animal model}

Basic anesthesia was induced by intramuscular injection of ketamine $(100 \mathrm{mg} / \mathrm{kg})$ and xylazine $(5 \mathrm{mg} / \mathrm{kg})$ and maintained by inhalation of isoflurane (2-3\% in oxygen, $3 \mathrm{~L} / \mathrm{min})$. Three VX2 tumors were implanted in the hind limbs of New Zealand White rabbits (Covance, Princeton, NJ, USA) and harvested when they reached approximately $3 \mathrm{~cm}$ in diameter as previously described ${ }^{17}$. Once harvested, small tumor fragments were sectioned for percutaneous ultrasound-guided tumor implantation via a biopsy needle into the liver ${ }^{17}$. Twelve additional adult New Zealand white male rabbits weighing $3.0-3.5 \mathrm{~kg}$ were anesthetized. Harvested tumor fragments (around $1 \mathrm{~mm}^{3}$ ) were implanted into the left medial and left lateral liver lobes. Tumors were allowed to grow for 7-10 days until the longest diameter reached $>10 \mathrm{~mm}$ by MRI measurement.

\section{IRE procedure}

A BTX Electroporator (ECM830; Harvard Apparatus, Holliston, MA) function generator and a parallel two-electrode array were used for all rabbit IRE procedures. The electrode array (each electrode was $25 \mathrm{~mm}$ long, with a diameter of $0.4 \mathrm{~mm}$ ) was inserted through a plastic block to maintain 5- or 10-mm spacing between the two parallel needles. 
Under anesthesia, baseline imaging was performed, and a mini-laparotomy was then conducted to access the tumor in the left liver lobe. The bipolar IRE electrode array was positioned with the two needles straddling the center of the left medial lobe tumor mass along the longest tumor diameter and then IRE pulse train was delivered. Eight pulses of $100-$ usec, $2000 \mathrm{~V} / \mathrm{cm}$ square wave pulses were applied with 100 -msec spacing ${ }^{18}$. After IRE tumor ablation, the abdominal incisions were closed.

\section{MR imaging and analysis}

MRI scans were carried out at baseline and immediately post-IRE by using a 3.0-T clinical MR imaging unit (Magnetom Skyra; Siemens Medical Solutions, Erlangen, Germany) with a clinical fifteen-element knee array coil. The acquisition protocol included axial T1weighted (T1W) gradient echo (GRE) imaging (TR: 200 ms; TE: 2.93 ms; slice thickness, $2 \mathrm{~mm}$; flip angle, $70^{\circ}$; field of view, $180 \mathrm{~mm} \times 180 \mathrm{~mm}$ ), axial T2-weighted (T2W) turbo spin echo (TSE) imaging (TR: due to respiratory gating approx. 4000 ms; TE: 39 ms; slice thickness, $2 \mathrm{~mm}$; flip angle, $150^{\circ}$; field of view, $180 \mathrm{~mm} \times 180 \mathrm{~mm}$ ), and DWI (TR: 1300 ms; TE: $65 \mathrm{~ms}$; slice thickness, $2 \mathrm{~mm}$; flip angle, $90^{\circ}$; field of view, $153 \times 124$; $\mathrm{b}$ values $=$ $0,400,800$, and $\left.1200 \mathrm{~s} / \mathrm{mm}^{2}\right)$.

ADC maps were generated offline by using Matlab (MathWorks, Natick, MA). Pre- and post-IRE MR images were reviewed by two radiologists together by consensus. 2D region-of-interests (ROIs) were manually drawn to cover the whole area of each tumor on the ADC maps in the axial view, using T2W and DW images as anatomic references. The 
changes in tumor $A D C$ values after IRE were calculated by using the following equation: $\triangle A D C=A D C_{\text {post-IRE }}-A D C_{\text {pre-IRE, }}$, where $A D C_{\text {pre-IRE }}$ indicates the tumor $A D C$ before IRE, and $A D C_{\text {post-IRE }}$ indicates the tumor $A D C$ after IRE.

\section{Histologic exam}

All rabbits were euthanized immediately after post-IRE MRI examination. The orientation of two tissue sections was chosen to best match MR acquisition geometry and sent them to \#\#\#\#\#\#\#\# Pathology Core for hematoxylin-eosin (HE) staining to assess necrosis area, and terminal deoxynucleotidyl transferase dUTP nick end labeling (TUNEL) staining to assess cell apoptosis. Resulting histologic slides were reviewed by a pathologist from the Pathology Core with more than 10 years of experience in gastrointestinal oncology. All quantitative measurements were performed with a pathologist assistance.

Quantification was performed by counting positive cell populations in randomly sampled regions by using the TissueFAXS system (TissueGnostics, Los Angeles, CA) at a high magnification. The Apoptosis Index (Al) is defined as a percentage of apoptotic cells and bodies per all tumor cells ${ }^{19}$.

\section{Statistical analysis}

All statistical analyses were implemented by using a statistical software package (SPSS, version 19; SPSS, Chicago, IL). Data were presented as means \pm standard deviations. 
The independent t-test was used to compare $A D C$ values and $\triangle A D C$ measurements between untreated tumors and IRE-treated tumors before and after IRE. The paired ttests were used to compare ADC values between baseline and after IRE treatment obtained in untreated tumor and IRE-treated tumors, respectively. Finally, the differences of apoptosis index between untreated tumors and IRE-treated tumors was analyzed using the independent t-test. $P<0.05$ was considered to be significant.

\section{RESULTS}

Eleven rabbits implanted with VX2 tumor cells developed liver tumors. One rabbit was excluded because it died during MR scanning after IRE procedure. Ten rabbits were included in this study. One tumor per rabbit underwent IRE treatment, while the other tumor served as untreated control.

\section{DW-MRI results}

All tumors were successfully visualized by MRI. The untreated tumor appears hyperintense on both $T 2 W$ images and $D W$ images and shows a relatively low $A D C$ value at baseline and post IRE MR scans. At baseline, the treated tumor showed hypointense signal intensity on T1W images (Figure $1 \mathrm{~A}$ ), on T2W image (Figure $1 \mathrm{~B}$ ) and on DW image (Figure 1 C), with decreased ADC value (Figure $1 \mathrm{D}$ ). Immediately after IRE, the IRE-treated tumor showed heterogeneous signal intensities on T1W (Figure 2 A), T2W (Figure 2 B) and DW images (Figure 2 C), with increased ADC value (Figure 2 D). 
The baseline ADC values averaged among all the rabbits were $(0.91 \pm 0.12) \times 10^{-3} \mathrm{~mm}^{2} / \mathrm{s}$ for IRE-treated tumors $(n=10)$ and $(0.84 \pm 0.18) \times 10^{-3} \mathrm{~mm}^{2} / \mathrm{s}$ for untreated tumors $(\mathrm{n}=$ 5). Immediately after IRE treatment, the ADC values were $(1.08 \pm 0.15) \times 10^{-3} \mathrm{~mm}^{2} / \mathrm{s}$ for IRE-treated tumors $(n=10)$ and $(0.88 \pm 0.19) \times 10^{-3} \mathrm{~mm}^{2} / \mathrm{s}$ for untreated tumors $(n=5)$. There were statistically significant differences in ADC between untreated and treated tumors after IRE $(P=0.042)$, and between baseline and post-IRE scans in treated tumors $(P=0.001)$. There was no statistically significant difference in ADC values between untreated and treated tumors at baseline $(P=0.427)$, or between baseline and post-IRE in untreated tumor $(P=0.624)$ (Figure $3 \mathrm{~A}) . \triangle \mathrm{ADC}$ was significantly higher in treated tumors compared to untreated tumors $(P=0.049)$ (Figure $3 \mathrm{~B})$.

\section{Histology results}

All rabbits were euthanized immediately after post-IRE MRI examination for histologic examinations. Necrotic area of the tumors was identified on HE-stained slices immediately after IRE. HE slices showed larger percentages of necrotic area in treated tumors (Figure 4 A) compared with untreated tumors (Figure 4 B). Then, TUNEL-stained slides were used to assess cell apoptosis in IRE treated tumors (Figure 5 A) and untreated tumors (Figure 5 B). Al measurements were statistically significantly different between IRE-treated tumors $(18.1 \pm 4.3 \%)$ and untreated tumors $(6.6 \pm 1.7 \%)$ (Figure 5 C) $(P<0.001)$. 


\section{DISCUSSION}

As with all forms of ablation, early assessment of objective response to IRE treatment is important as all tumor tissue may not be completely ablated ${ }^{2,18,20}$. This study demonstrated the potential for using DWI to evaluate the early treatment response of liver tumor in a rabbit model. ADC values were increased in treated tumors immediately after IRE procedure.

The ability of imaging methods to accurately assessment response of electroporation is useful for proper evaluation of treatment effectiveness. Previous studies have evaluated the role of $\mathrm{MRI}$ in assessing the response to IRE response in liver tissues ${ }^{2,7,9,10}$, in which contrast-enhanced MRI is the most widely used technique ${ }^{7,9,10}$. Contrast-enhanced MRI with T1-weighted gradient-recalled echo (GRE) is particularly effective for differentiating treated from untreated tissue zones after ablation treatment ${ }^{7}$. However, contrastenhanced MRI is always performed based on the utilize of Gadolinium. While the product of Gadolinium-based contrast agents can accumulate in a range of tissues and organs (skin, bone, liver, kidney, muscle, brain, et al) albeit in very low concentrations, which then cause pathological changes of this tissues ${ }^{21,22}$. Therefore, further imaging modalities, rather than Gadolinium-based imaging techniques, are required for accurately assessment of IRE ablation.

DWI has been proposed as a potential early imaging technique for cancer treatment, since it can provide information about tumor viability, cellularity and vascular distribution both at baseline and post-therapy ${ }^{23-25}$. Changes in ADC values can be promising 
indicators of therapeutic responses at early stages and may change before anatomic changes occur ${ }^{24,26}$. Furthermore, DWI can effectively monitor tumor status after transarterial chemoembolization therapy, as ADC values can distinguish viable tumor areas from necrotic areas ${ }^{15,26}$. However, only limited published investigations have demonstrated the possible application of DW-MRI to assess treatment response to IRE ${ }^{12}$. Herein, DWI findings were performed to quantitatively characterize the lesion using ADC values immediately after IRE treatment. The IRE-treated tumors showed a statistically significant increase in ADC values immediately after IRE, possibly due to increased tumor cell membrane permeability from the high-voltage pulses. IRE induces cell membrane damage and cellular apoptosis using high-voltage electrical fields ${ }^{27}$ that alters the cellular transmembrane potential and disrupts the lipid bilayer to create "nanopores" in the cell membrane ${ }^{1,10,12}$. Then, local fluid will accumulate with subsequent fluid build-up after rapid extravasation into the treated tissue areas. This alteration of microstructure results in less hindrance to the motion of water molecules, which may increase ADC values ${ }^{14,28,29}$.

$A D C$ was calculated rather than to use more advanced diffusion models for the sake of simplicity and translational potential. More advanced DWI methods, such as intravoxel incoherent motion or diffusion kurtosis imaging ${ }^{30,31}$, are not widely used in liver imaging and require more complicated acquisition and processing protocols that are not easy to implement in clinical settings. The current study design was focused upon clinical translation. All imaging methods proposed were approved by the FDA and these results are directly applicable to human studies. 
A consensus on the IRE treatment protocol (number, duration and intensity of pulses) has not been reached yet. In this study, a total of eight $100-$ usec, $2000 \mathrm{~V} / \mathrm{cm}$ square wave pulses were applied with 100-msec spacing. These specific IRE timing parameters were chosen to achieve IRE ablation of tumor tissue based on previous studies ${ }^{18,29}$.

This study has the following limitations: First, although histological measurements were highly correlated with ADC measurements of the IRE-treated tumor, a variable level of discrepancy between these measurements was found. Additional more systematic study of pathology changes related to ADC findings may be important, but difficult given the large gap between histology and in vivo MR spatial resolution and complexities associated with in vivo image to ex vivo slide co-registration. Second, potential bias might be presented when drawing the exact contours of the tumors because it was not blinded to the radiologists whether the tumor was treated. Finally, imaging was performed only at a single time point immediately after IRE treatment. Further studies should be undertaken to establish the long-term prognostic value of these DWI metrics, correlating early DWI measures with end-point histology and/or survival data.

In conclusion, this study demonstrated that DWI can be used to quantitatively evaluate treatment response in liver tumors immediately after IRE procedure in an animal model. These findings support that DWI measurements of IRE-induced tumor responses might 
be served as an innovative new approach for early detection of IRE therapy response in clinical applications. 


\section{REFERENCES}

1. Ruarus AH, Vroomen LGPH, Puijk RS, et al. Irreversible Electroporation in Hepatopancreaticobiliary Tumours. Can Assoc Radiol J 2018; 69: 38-50.

2. Zhang Y, Guo Y, Ragin AB, et al. MR Imaging to Assess Immediate Response to Irreversible Electroporation for Targeted Ablation of Liver Tissues: Preclinical Feasibility Studies in a Rodent Model. Radiology 2010; 256: 424-432.

3. Charpentier KP, Wolf F, Noble L, et al. Irreversible electroporation of the liver and liver hilum in swine. HPB (Oxford) 2011; 13: 168-173.

4. Distelmaier M, Barabasch A, Heil P, et al. Midterm Safety and Efficacy of Irreversible Electroporation of Malignant Liver Tumors Located Close to Major Portal or Hepatic Veins. Radiology 2017; 285: 1023-1031.

5. $\mathrm{Li} \mathrm{X}, \mathrm{Xu} \mathrm{K}, \mathrm{Li} \mathrm{W}$, et al. Immunologic Response to Tumor Ablation with Irreversible Electroporation. PLoS One 2012; 7: e48749.

6. Al-Sakere B, Bernat C, André F, et al. A Study of the Immunological Response to Tumor Ablation with Irreversible Electroporation. Technol Cancer Res Treat 2007; 6: 301-305.

7. Guo Y, Zhang Y, Nijm GM, et al. Irreversible Electroporation in the Liver: Contrast-enhanced Inversion-Recovery MR Imaging Approaches to Differentiate Reversibly Electroporated Penumbra from Irreversibly Electroporated Ablation Zones. Radiology 2011; 258: 461-468.

8. Figini M, Wang X, Lyu T, et al. Preclinical and clinical evaluation of the liver tumor irreversible electroporation by magnetic resonance imaging. Am J Transl Res 2017; 9: 580-590. 
9. Felker ER, Dregely I, Chung DJ, et al. Irreversible Electroporation: Defining the MRI Appearance of the Ablation Zone With Histopathologic Correlation in a Porcine Liver Model. Am J Roentgenol 2017; 208: 1141-1146.

10. Padia SA, Johnson GE, Yeung RS, et al. Irreversible Electroporation in Patients with Hepatocellular Carcinoma: Immediate versus Delayed Findings at MR Imaging. Radiology 2015; 278: 285-294.

11. Jing C, L. DB, J. DC, et al. Monitoring prostate thermal therapy with diffusion-weighted MRI. Magn Reson Med 2008; 59: 1365-1372.

12. Granata V, de Lutio di Castelguidone E, Fusco R, et al. Irreversible electroporation of hepatocellular carcinoma: preliminary report on the diagnostic accuracy of magnetic resonance, computer tomography, and contrast-enhanced ultrasound in evaluation of the ablated area. Radiol Med 2016; 121: $122-131$.

13. Saito K, Tajima Y, Harada TL. Diffusion-weighted imaging of the liver: Current applications. World J Radiol 2016; 8: 857-867.

14. Lewis HL, Ghasabeh MA, Khoshpouri P, et al. Functional hepatic imaging as a biomarker of primary and secondary tumor response to loco-regional therapies. Surg Oncol 2017; 26: 411-422.

15. Gluskin JS, Chegai F, Monti S, et al. Hepatocellular Carcinoma and Diffusion-Weighted MRI: Detection and Evaluation of Treatment Response. J Cancer 2016; 7: 1565-1570.

16. Shenoy-Bhangle A, Baliyan V, Kordbacheh H, et al. Diffusion weighted magnetic resonance imaging of liver: Principles, clinical applications and recent updates. World J Hepatol 2017; 9: $1081-1091$.

17. White SB, Chen J, Gordon AC, et al. Percutaneous Ultrasound Guided Implantation of VX2 for Creation of a Rabbit Hepatic Tumor Model. PLoS One 2015; 10: e0123888. 
18. Wang X, Su Z, Lyu T, et al. 18F-FDG PET Biomarkers Help Detect Early Metabolic Response to Irreversible Electroporation and Predict Therapeutic Outcomes in a Rat Liver Tumor Model. Radiology 2017; 287: 137-145.

19. Lyu T, Wang X, Su Z, et al. Irreversible electroporation in primary and metastatic hepatic malignancies: A review. Medicine (Baltimore) 2017; 96: e6386.

20. Sugimoto K, Moriyasu F, Saito K, et al. Multimodality imaging to assess immediate response following irreversible electroporation in patients with malignant hepatic tumors. $J$ Med Ultrason 2017; 44: 247-254.

21. Kanda T, Fukusato T, Matsuda M, et al. Gadolinium-based Contrast Agent Accumulates in the Brain Even in Subjects without Severe Renal Dysfunction: Evaluation of Autopsy Brain Specimens with Inductively Coupled Plasma Mass Spectroscopy. Radiology 2015; 276: 228-232.

22. Hoggard N, Roditi GH. T(1) hyperintensity on brain imaging subsequent to gadolinium-based contrast agent administration: what do we know about intracranial gadolinium deposition? $\mathrm{Br} J$ Radiol 2017; 90: 20160590.

23. Corona-Villalobos CP, Halappa VG, Bonekamp S, et al. Functional Magnetic Resonance Imaging Response of Targeted Tumor Burden and Its Impact on Survival in Patients With Hepatocellular Carcinoma. Invest Radiol 2015; 50: 283-289.

24. Schmeel FC, Simon B, Sabet A, et al. Diffusion-weighted magnetic resonance imaging predicts survival in patients with liver-predominant metastatic colorectal cancer shortly after selective internal radiation therapy. Eur Radiol 2017; 27: 966-975.

25. Fusco R, Petrillo M, Granata V, et al. Magnetic Resonance Imaging Evaluation in Neoadjuvant Therapy of Locally Advanced Rectal Cancer: A Systematic Review. Radiol Oncol 2017; 51: $252-$ 262. 
26. Qian T, Chen M, Gao F, et al. Diffusion-weighted magnetic resonance imaging to evaluate microvascular density after transarterial embolization ablation in a rabbit VX2 liver tumor model. Magn Reson Imaging 2018; 32: 1052-1057.

27. Bulvik BE, Rozenblum N, Gourevich S, et al. Irreversible Electroporation versus Radiofrequency Ablation: A Comparison of Local and Systemic Effects in a Small-Animal Model. Radiology 2016; 280: 413-424.

28. Appelbaum L, Ben-David E, Sosna J, et al. US Findings after Irreversible Electroporation Ablation: Radiologic-Pathologic Correlation. Radiology 2012; 262: 117-125.

29. Zhang Y, White SB, Nicolai JR, et al. Multimodality Imaging to Assess Immediate Response to Irreversible Electroporation in a Rat Liver Tumor Model. Radiology 2014; 271: 721-729.

30. Shanshan L, Feng S, Kaikai W, et al. Intravoxel Incoherent Motion Diffusion-weighted MR Imaging for Early Evaluation of the Effect of Radiofrequency Ablation in Rabbit Liver VX2 Tumors. Acad Radiol. Epub ahead of print 9 May 2018. DOI: 10.1016/j.acra.2018.01.010.

31. Goshima S, Kanematsu M, Noda Y, et al. Diffusion Kurtosis Imaging to Assess Response to Treatment in Hypervascular Hepatocellular Carcinoma. Am J Roentgenol 2015; 204: W543-W549. 


\section{FIGURES}

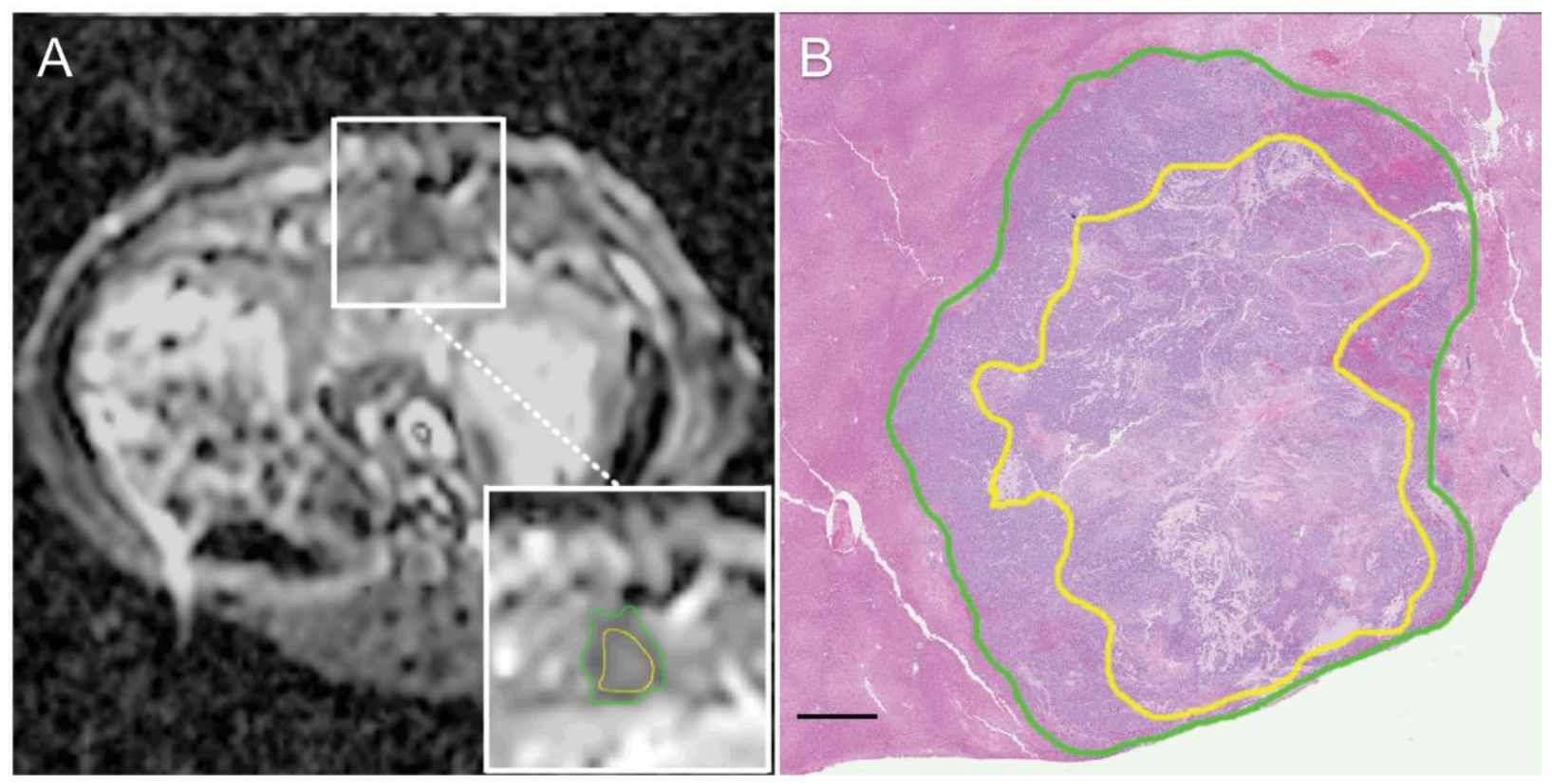

Figure 1 Representative axial T1W images $(\mathbf{A})$, axial T2W images $(\mathbf{B})$, DW images $(b=$ $\left.800 \mathrm{~s} / \mathrm{mm}^{2}\right)(\mathbf{C})$ and tumor ADC maps (color-coded and superimposed on T2W image) (D) obtained at Pre-IRE. The tumor was hypointense on T1W images, and hyperintense on T2W images and DW images before IRE. ADC maps showed low ADC values. 

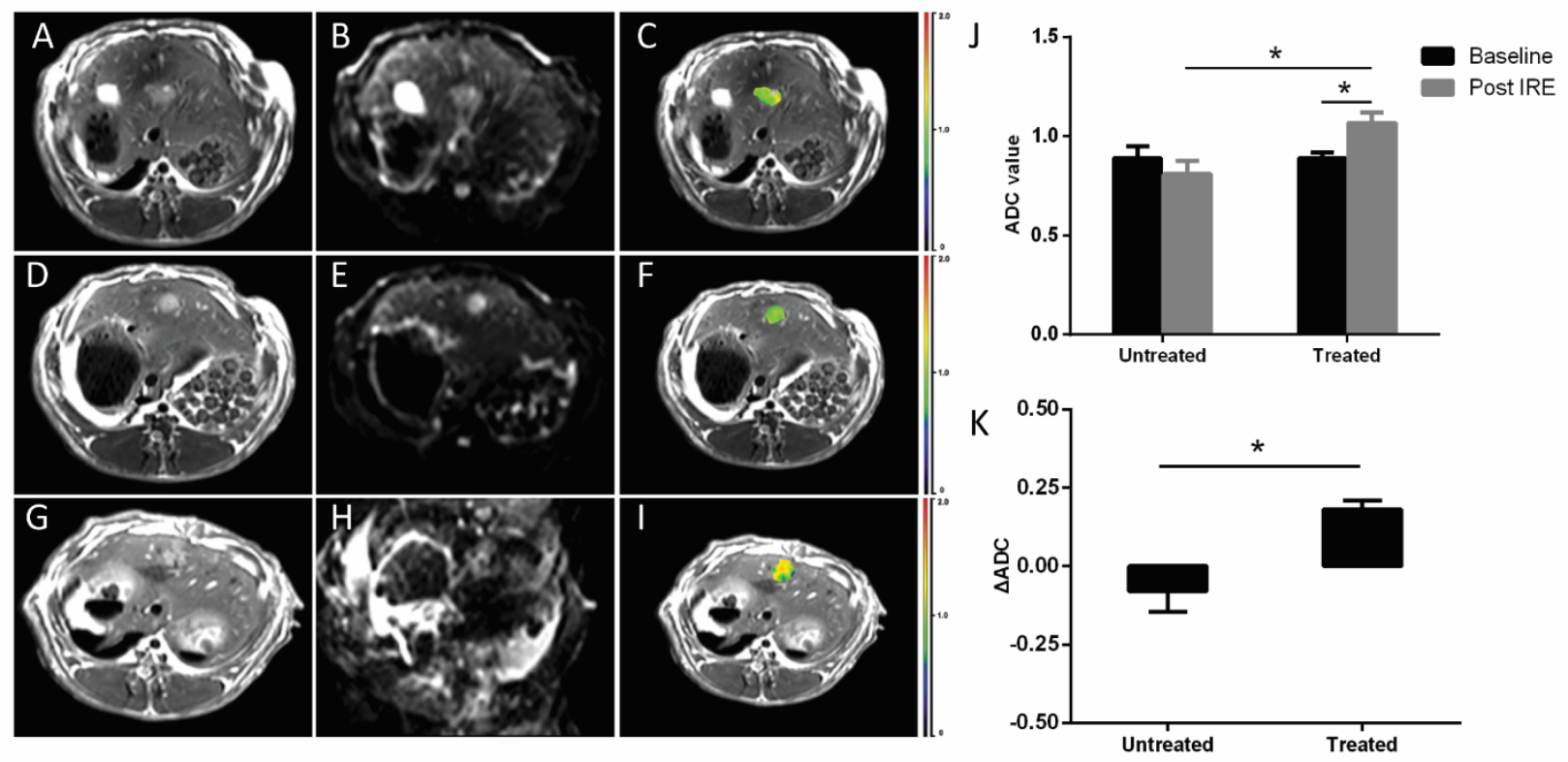

Figure 2 Representative axial T1W images (A), axial T2W images (B), DW images (b = $\left.800 \mathrm{~s} / \mathrm{mm}^{2}\right)(\mathbf{C})$ and tumor ADC maps (color-coded and superimposed on T2W image) (D) obtained at Post-IRE. The tumor was hypointense on T1W images, and heterogeneously hyperintense on T2W images and DW images post IRE. ADC maps showed the increase in ADC values. 

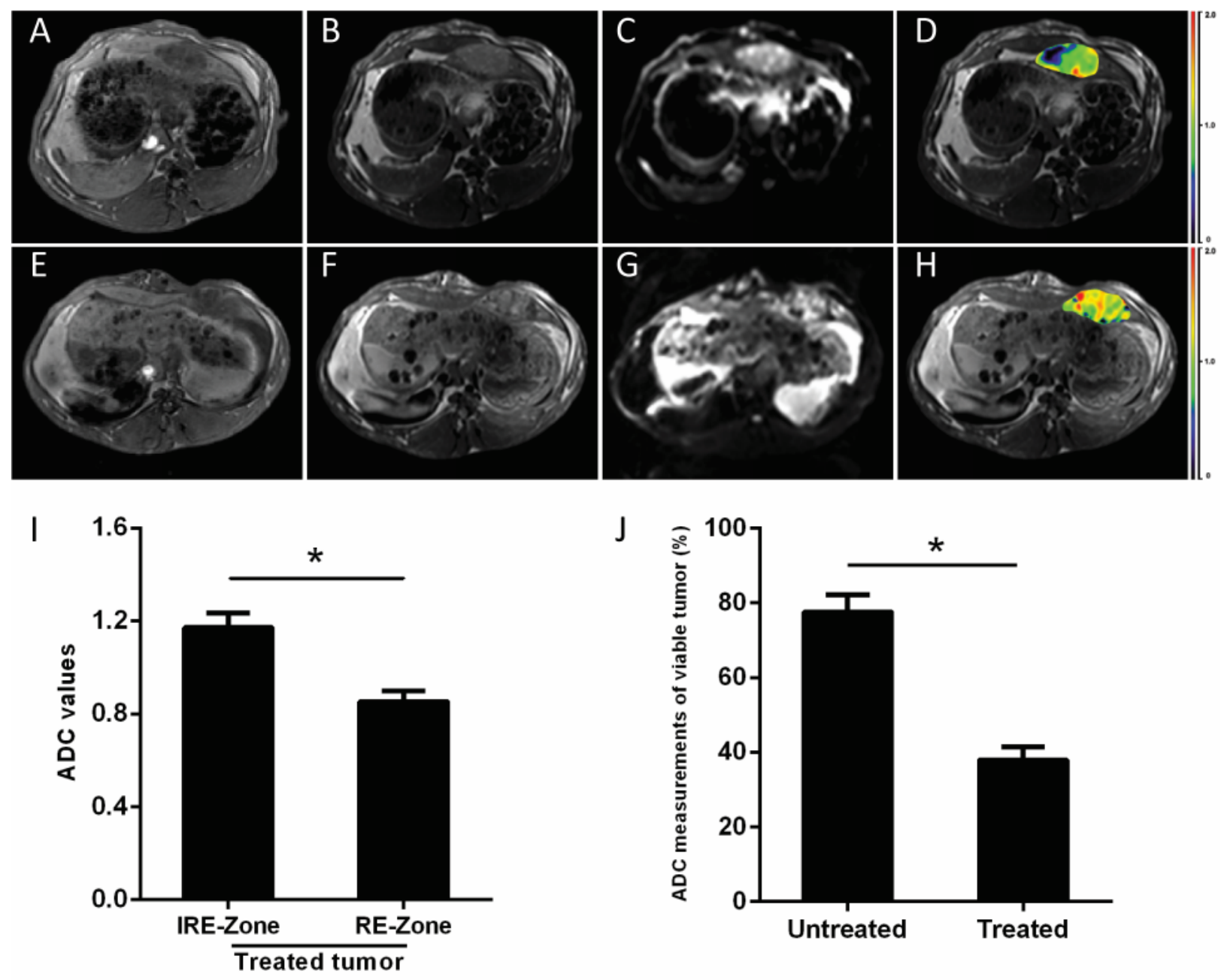

Figure 3 The bar chart $(\mathbf{A})$ shows a statistically significant difference in ADC values between untreated tumors and treated tumors after IRE $(P=0.042)$, and between baseline and immediately after IRE with IRE-treated tumors $(P=0.001)$. The box plot $(B)$ shows a statistically significant difference in $\triangle A D C$ between treated and untreated tumors $(P=0.049)$. 

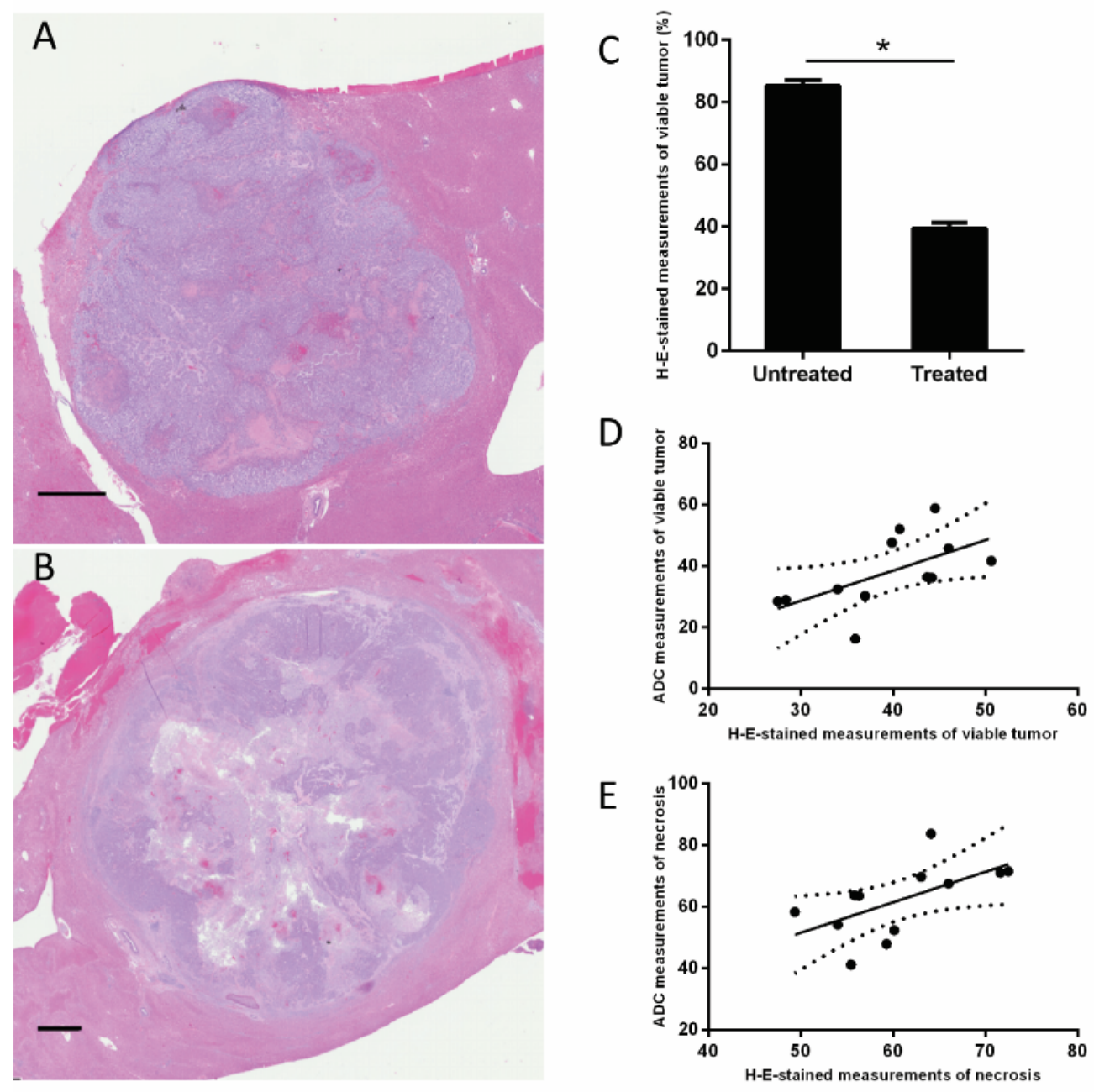

Figure 4 Representative photomicrographs (HE stained) of treated (A) and untreated (B) tumor immediately after IRE (scale bar $=2.5 \mathrm{~mm}$ ). Treated tumor showed larger percentages of necrotic area than untreated tumor. 



Figure 5 Tumor TUNEL staining and quantitative measurement of apoptotic tumor cells. Photomicrographs (TUNEL stain) in treated tumor (A) and untreated tumor (B) immediately after IRE show decreasing levels of apoptosis (scale bar = 20 um). The bar charts show statistically significant difference in Al measurements between treated and untreated tumors $(P<0.001, \mathbf{C})$. 